\title{
Digital Image Restoration via Neural Networks Design
}

\author{
Ahmed Abdualah Aburas, Abdussalam Alhadi Addeeb \\ Elmergeb University, ECE Department, Khoms - Libya \\ DOI: 10.29322/IJSRP.11.09.2021.p11740 \\ http://dx.doi.org/10.29322/IJSRP.11.09.2021.p11740
}

\begin{abstract}
The idea of this paper is to link artificial neural networks (ANN) and image filters to restore corrupted images. The ANN predicts the density of the noise in the input image. Then, the appropriate variables for filter (window) determines the ratio of the expected density of the noise.

This process makes the filters adaptive and self-modifying to fit the noise densities of distorted image. The proposed restoration techniques include adaptive median filter (AMF) and adaptive switched median filter (ASMF) for salt and pepper noise. Furthermore, adaptive gaussian filter (AGF) is proposed for Gaussian noise. The performance of the previous filters is compared with the classic adaptive median filter (CAMF). Both objective measures and subjective evaluation have been utilized in assessing the output images. The eye is to visualize the image details and its intelligence subjectively. While the quality metrics: mean square error (MSE), peak signal-to-noise ratio (PSNR) and image enhancement factor (IEF) are used to measure the performance of proposed system objectively.
\end{abstract}

Index Terms- Image denoising, adaptive filters, neural networks

\section{INTRODUCTION}

The image is subjected to a variety of undesired signals (distortion), including noise, blur, and the loss of some image data, such as when the image is exposed to spots or fractures, which causes some key information to be lost. The image restoration field is focused with estimating and reconstructing the most probable original image from a noisy and blurred version for its efficacy. The idea of image restoration lies in performing inverse operations to those imperfection causes during the formation and/or transmission of the desired image in all of these operations and processes [1][2].

A new algorithm (adaptive median filter) for the removal of impulse noise from highly corrupted images was introduced. It presents a simple, yet efficient way to remove impulse noise from digital images. This novel method entails detecting the impulse noise from the image optimally. It is based on the intensity values, where the pixels are roughly divided into two classes, which are "noise-free pixels" and "noisy pixels". The next stage of the method is to eliminate the impulse noise from the image [3].

Authors in [4], have proposed a new kind of weighted median filtering algorithm that is used for image de-noising. The new algorithm initially decides commotion focuses in the picture through uproarious pixels recognition, then, at that point changes the size of sifting window adaptively as per the quantity of clamor focuses in the window. The pixel points in the separating window are gathered adaptively by specific standards and are given comparing weight to each gathering of pixel points dependent on similitude. At long last, the clamor distinguished are separating treated through means of weighted median filtering algorithm.

In [5], Akkoul, et al, have proposed a new adaptive switching median (ASWM) filter for removing impulse noise from corrupted images. The unique feature of ASWM is that, unlike a traditional Switching Median filter, it does not require an a priori threshold. Instead, Threshold is calculated locally from the intensity values of image pixels in a sliding window. For random-valued impulse noise, it performs better in terms of PSNR and MAE than many other median filter variants for random impulse noise.

In [6], Jiang and Shen, have proposed an effective adaptive median filter algorithm for removing salt \& pepper noise in images. It solved the problem of the simplified pulse coupled neural network model in image filtering. The simplified model is proved to fail to detect pepper noise using the method of reduction absurdum and the model have been improved using the method of divide and rule. Finally, the adaptive median filter algorithm is achieved by detecting the pollution level of the image.

In [7], the proposed artificial neural network based weighted median filter (ANNWMF) extracts features from a sliding kernel of the corrupted image. ANNWMF classifies the features into noisy and non-noisy pixels, and adjusts the corrupted and noncorrupted pixels. ANNWMF which is a computational model based on the structure and functions of biological neural networks along with filtering techniques has been observed to lower mean square error (MSE) that increases the peak-signal-tonoise ratio (PSNR) for high density of noise.

In [8], the authors have proposed a candidate method for classification of multi class object images which are corrupted by Gaussian noise. A wavelet transform based de-noising scheme by thresholding the wavelet coefficients namely neigh shrink will be utilized to eliminate too many wavelet coefficients that might contain noise signal and selecting useful coefficients. This work is anticipated to display robust proposed method of multiclass object classification over the spatial domain denoising and feature extraction method for classification.

The idea of the proposed system in this project, is to link ANNs and adaptive filters employed to restore digital images. The ANN predicts the density of the noise in the input image. 
Then, the appropriate variables for an adaptive filter (window) specifies the ratio of the anticipated density of the noise. This process makes the adaptive filter adapt and self-modify to fit the noise densities of impaired image.

\section{FILTERS}

\section{A. Median Filter $(M F)$}

The MF is a noise-reduction method that employs nonlinear digital filtering. Noise reduction is a common pre-processing procedure used to improve the outcomes of later processing. The filters can be presented as follows:

$$
\hat{f}(i, j)=\operatorname{median}_{(s, t) \in S_{x y}}\{g(s, t)\}
$$

And switched median filter (SMF).

$$
\hat{f}_{1}(i, j)=\left\{\begin{array}{lc}
S(i, j) & \text { for } 0<S(i, j)>255 \\
\hat{f}(i, j) & \text { otherwise }
\end{array}\right.
$$

Where $(s, t) \in \mathrm{W}$, w represents a neighborhood defined by the user, centered around location $(i, j)$ in the image[9][10].

\section{B. Gaussian Filter $(G F)$}

Gaussian filter is a non-uniform low pass filter. Gaussian filter is used to blur images and remove noise and detail. It does not remove salt \& pepper noise effectively. The 2-D filters used in image processing can be presented as follows:

$$
P(z)=\frac{1}{\sqrt{2 \pi} \sigma} e^{-(z-\mu)^{2} / 2 \sigma^{2}}
$$

Where $\sigma$ is the standard deviation of the distribution. The distribution is assumed to have a mean $\mu$ of zero. We need to discretize the continuous Gaussian functions to store it as discrete pixels [9][10].

\section{Classic Adaptive Median filter (CAMF)}

The AMF is designed to get rid of the problems that take place with the Standard Median Filter. The essential distinction between the two filters is that in the AMF, the size of the window surrounding each pixel is variable. This variation relies on the median of the pixels in the present window. If the median value is an impulse, then the size of the window is expanded.

The adaptive filter operates on a WinMax rectangular area. During the filtering operation, the adaptive median filter modifies the size of WinMax based on the principles stated below. The filter's output is a single value that replaces the current pixel value at $(i, j)$, the point on which WinMax is now centered. The AMF operates on two steps, as shown below [3][6].

\section{$\rightarrow$ Step 1:}

Compute the Gmax , Gmed and Gmin of the window
If Gmax > Gmed > Gmin

go to step 2

Else increase the window size by 2

If (Window size $<=$ WinMax )

repeat level one

Else $F(i, j)=g(i, j)$

\section{$\rightarrow$ Step 2:}

If $G \max >g(i, j)>G \min$

$F(x, y)=g(i, j)$

Else $F(i, j)=$ Gmed

Where:

- Gmin: minimum value in Window.

- Gmax: maximum value in Window.

- Gmed: median of gray levels in Window.

- $\boldsymbol{g}(\boldsymbol{i}, \boldsymbol{j})$ : gray value of the image at $(i, j)$.

- WinMax: maximum allowed size of Window.

\section{THE PROPOSED ADAPTIVE FILTER BY ANN (PROPOSED SYSTEM)}

Figure 1 represents the adaptive filter algorithm of the ANN. Its input is the noisy image, which is passed to the neural network (NN). The NN estimates the noise density existed in the image and accordingly the filter will be ready to receive the distorted image and restore it. The filter works to recover the most optimum image that is closest to the original image.

The proposed adaptive filter by ANN is expected to show better results than standard adaptive filters in terms of quality and accuracy. Results of the proposed system will be presented in chapter 4 . The adaptive filter by ANN system is characterized by the pre-vision of the intensity of noise in digital images, that other adaptive filter without NN do not have. Furthermore, this system is characterized by less repetitive and excessive calculations, which affect the computation speed positively.

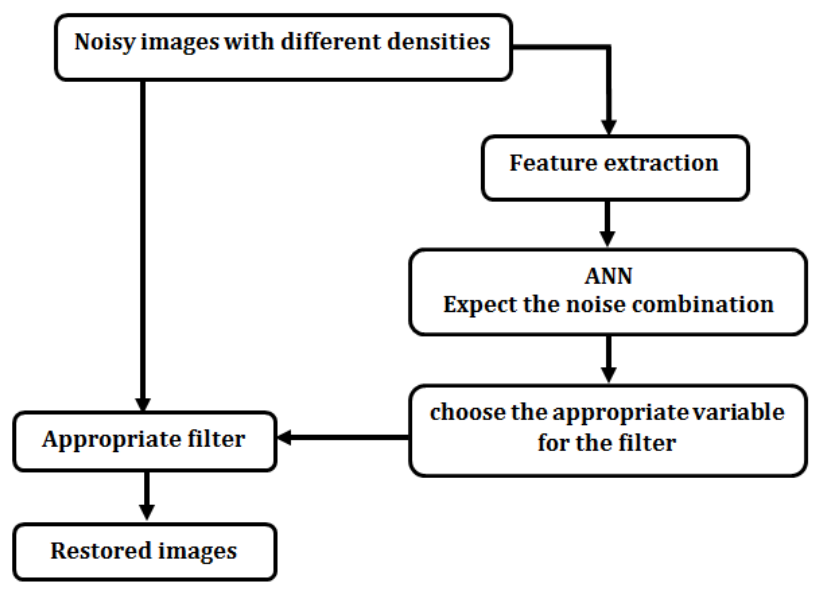

Figure 1: Adaptive filter by ANN (proposed system). 


\section{A. Feature extraction}

- Convert an image from gray to binary $(0,1)$.

- Take the average of every column in the image matrix, so that it becomes one row with a length of 256 elements.

- Convert the image array from row to column

- Apply the previous steps to each image with different noise intensity

- Put arrays unilateral in a single matrix to represent a complete data

- Each row of the input matrix corresponds to the output matrix and represents the value of the input noise.

\section{B. Neural Network Design}

The structure of the network utilized in this research is consisted of three layers $(1,6,256)$. These layers can be presented as follows.

Input Layer: Consists of 256 neural cells which represent the noisy image that be in a one sided matrix form as it is mentioned.

Single Hidden Layer: Consists of sixneural cells for the network of salt \& pepper noise. This is in addition to seven neurons for the Gaussian noise network.

Output Layer: It consists of one output, its values vary from 0 to 1 and it represents the noise density. The activation function (tansig) is used in the hidden layer for the Salt \& Pepper noise network and (Logsig) is used for Gaussian noise network. The linear function is used in the output layer and learning rate 0.1 for each of the two networks.

Figures 2 and 3 show the relationship between the errors and the number of runs needed to reach the required accuracy level such that the errors should be as low as possible. This can be achieved through testing it by a group of images. Images are classified into three groups : One for training equals $80 \%$, another for testing equals $10 \%$, and a third one for effectiveness of the training (validation) equals 10\%. These models requires (20 and 5) Epochs for the least possible error. This design is obtained after many trials. It is the most optimum model in terms of the results obtained and errors occur.

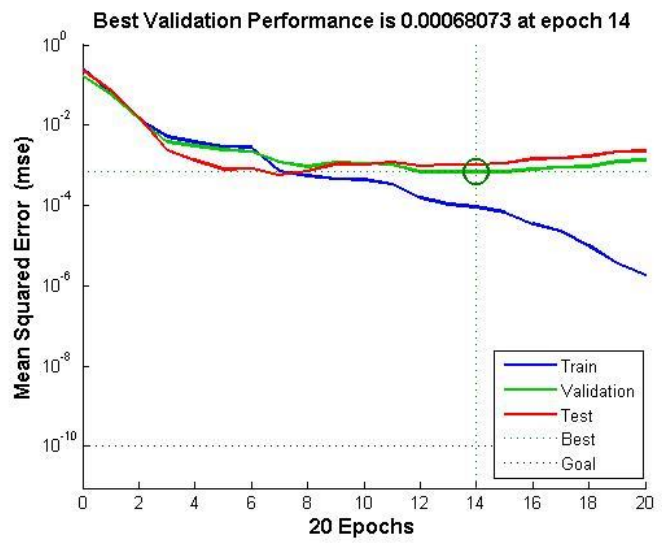

Figure 2: Performance of the training testing and validation of the neural network for salt \& pepper noise.

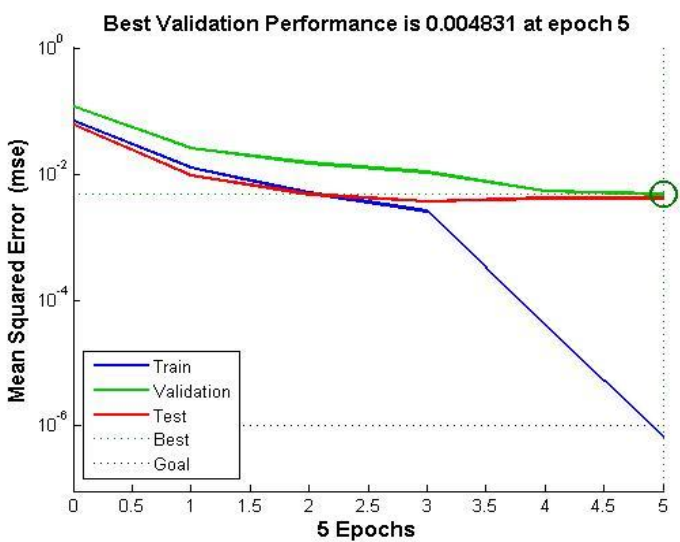

Figure 3: Performance of the training testing and validation of the neural network for gaussian noise.

\section{PERFormance MeAsures}

A. Mean square error (MSE)

$$
M S E_{2}=\frac{\sum_{i} \sum_{j}(O(i, j)-R(i, j))^{2}}{L^{*} W}
$$

\section{B. Peak signal-to-noise ratio (PSNR)}

$$
\operatorname{PSNR}(d B)=10 \log _{10}\left(\frac{255^{2}}{M S E}\right)
$$

\section{Image enhancement factor (IEF)}

$$
I E F=\frac{\sum_{i} \sum_{j}(N(i, j)-O(i, j))^{2}}{\sum_{i} \sum_{j}(R(i, j)-O(i, j))^{2}}
$$

Where:

$\mathrm{O}$ : original image

$\mathrm{R}$ : restored image

$\mathrm{N}$ : noisy image.

$\mathrm{L} * \mathrm{~W}$ : size of the image [11][12].

\section{RESUlTS}

Subjective and objective results of the proposed system are illustrated and discussed in this section.

\section{A. Results for Salt and Pepper Noise}

Two adaptive filters are used to restore images out of salt \& pepper noisy data. These filters are:

- Adaptive median filter (AMF).

- Adaptive switched median filter (ASMF).

The above filters are compared with classic adaptive median filter (CAMF). 
TABLE I: Noise prediction (salt \& pepper) for three different images.

\begin{tabular}{|c|c|c|c||}
\hline \multirow{2}{*}{ Noise density \% } & \multicolumn{3}{|c|}{ Noise Prediction } \\
\cline { 2 - 4 } & Cameraman & Flower & Moon \\
\hline $\mathbf{1 0}$ & 0.1434 & 0.1394 & 0.1374 \\
\hline $\mathbf{2 0}$ & 0.2010 & 0.1983 & 0.1979 \\
\hline $\mathbf{3 0}$ & 0.2455 & 0.2838 & 0.2574 \\
\hline $\mathbf{4 0}$ & 0.3897 & 0.3896 & 0.4182 \\
\hline $\mathbf{5 0}$ & 0.4985 & 0.5189 & 0.5007 \\
\hline $\mathbf{6 0}$ & 0.6025 & 0.5760 & 0.5712 \\
\hline $\mathbf{7 0}$ & 0.7018 & 0.7138 & 0.8023 \\
\hline $\mathbf{8 0}$ & 0.8622 & 0.8423 & 0.8493 \\
\hline $\mathbf{9 0}$ & 0.9308 & 0.9338 & 0.9523 \\
\hline $\mathbf{1 0 0}$ & 0.9812 & 0.9779 & 0.9632 \\
\hline
\end{tabular}

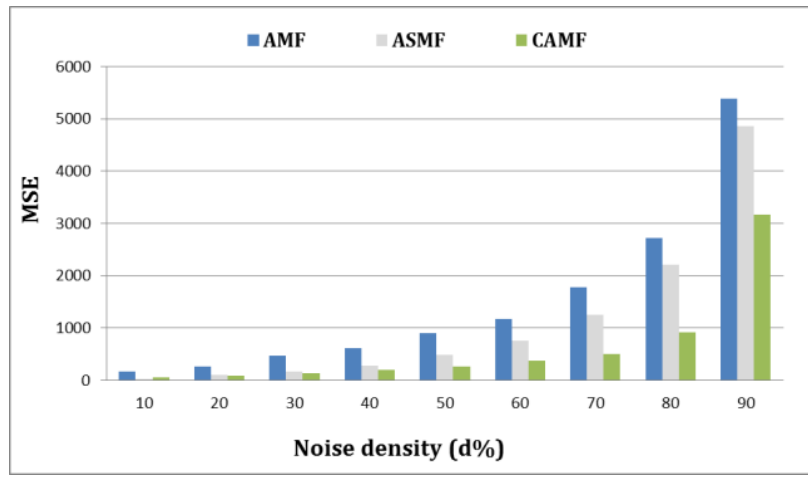

Figure 4: MSE versus of salt \& pepper noise.

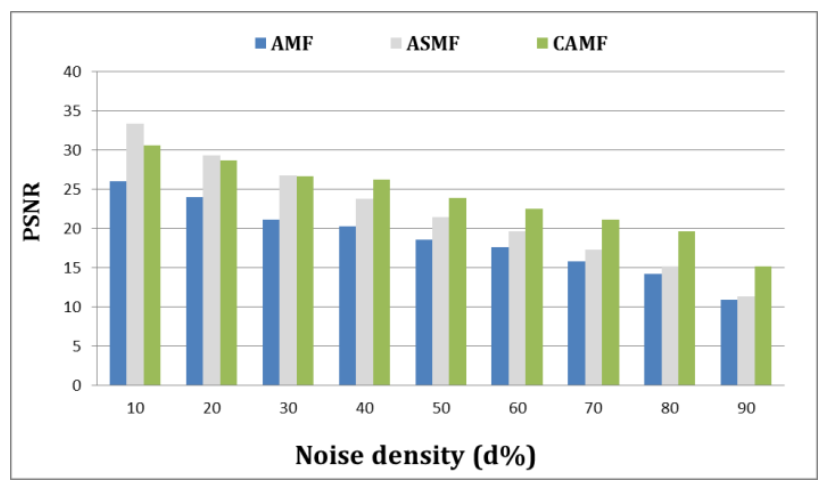

Figure 5: PSNR versus of salt \& pepper noise.

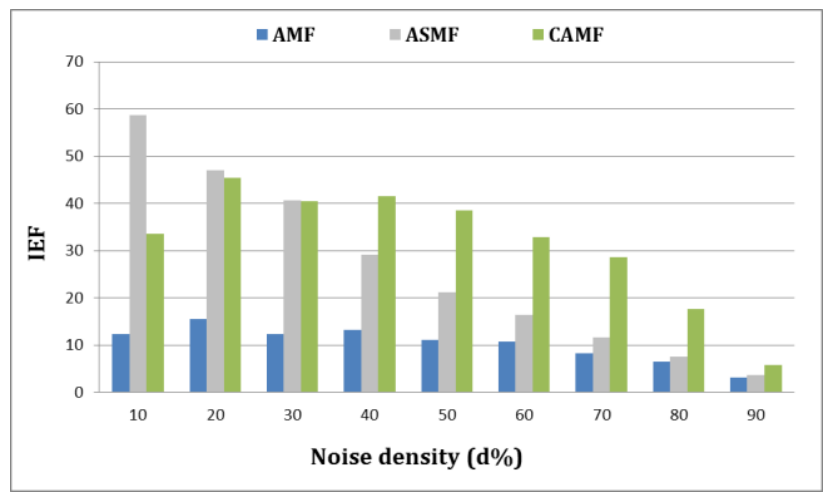

Figure 6: IEF versus of salt \& pepper noise.

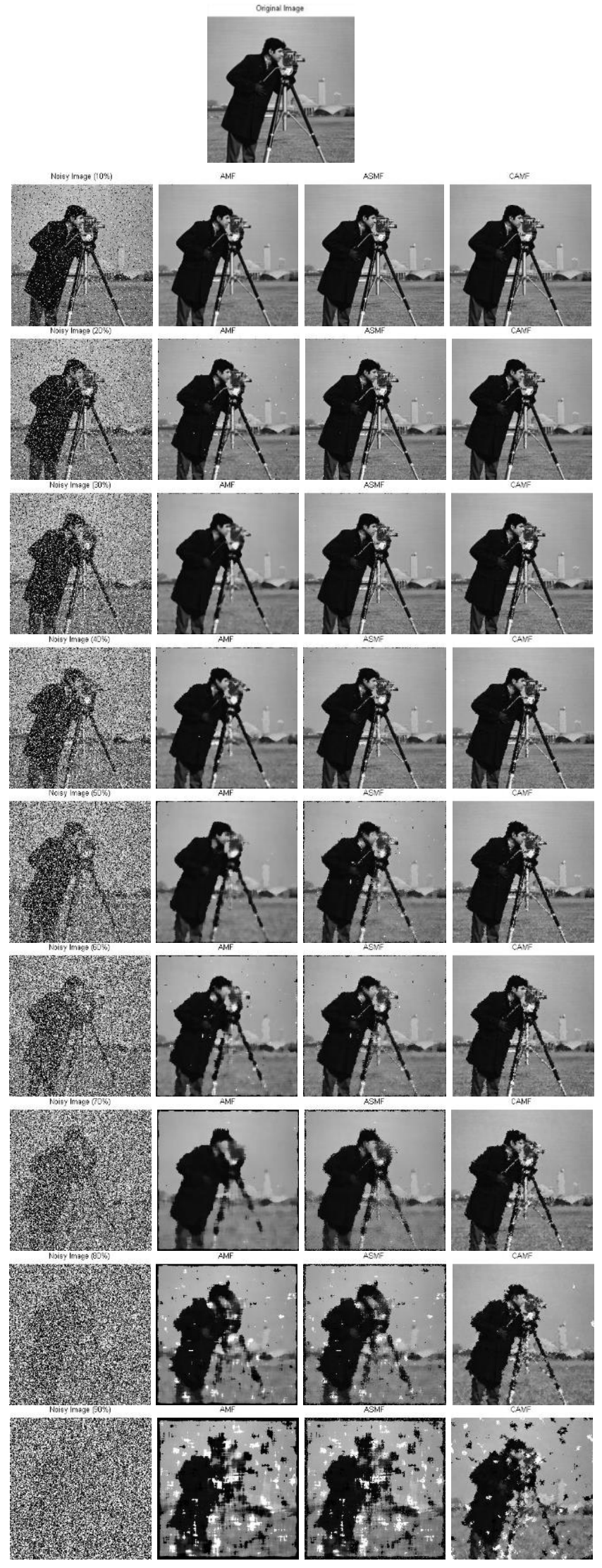

Figure 6: Processed results of various AFs for image corrupted by salt $\&$ pepper noise. 
In the noise density range of $10 \%-30 \%$, the ASMF filter has the highest average performance with MSE measure of 13.3703, PSNR measure of 29.8149 IEF measure of 43.4477. The second rank is for the CAMF filter that has an average performance of $\mathrm{MSE}=94.0042, \mathrm{PSNR}=28.6111$ and $\mathrm{IEF}=42.105 . \mathrm{AMF}$ filter comes last with $\mathrm{MSE}=300, \mathrm{PSNR}=23.7001$ and $\mathrm{IEF}=13.3703$. While in the noise density range of $40 \%-90 \%$, CAMF filter comes first with an average MSE $=901.092$, average PSNR= 21.4373 and average IEF $=27.4937$ followed by ASMF with an average performance of $\mathrm{MSE}=1642.157, \mathrm{PSNR}=18.0979$ and $\mathrm{IEF}=14.9278$. AMF filter comes last with the least performance of average measure, $\mathrm{MSE}=2093.924, \mathrm{PSNR}=16.2358$ and $\mathrm{IEF}=8.8328$.

ANN system performs extraordinary throughout the lower and medium range (less than $60 \%$ ), taking into consideration that the informative image is impaired gradually due to continual noise increase along the range. ASMF and CAMF filters have the advantage of avoiding blur and preserving edges more than AMF has.

\section{B. Results for Gaussian Noise}

There For Gaussian noise, two filters are used as well. These filters are:

- Adaptive median filter (AMF).

- Adaptive gaussian filter (AGF).

The above filters are compared with classic adaptive median filter (CAMF).

TABLE II: Noise prediction (gaussian) for three different images.

\begin{tabular}{|c|c|c|c||}
\hline \multirow{2}{*}{ Noise density \% } & \multicolumn{3}{|c|}{ Noise Prediction } \\
\cline { 2 - 4 } & Cameraman & Flower & Moon \\
\hline $\mathbf{1 0}$ & 0.1037 & 0.0081 & 0.0845 \\
\hline $\mathbf{2 0}$ & 0.2010 & 0.0575 & 0.3090 \\
\hline $\mathbf{3 0}$ & 0.2544 & 0.2838 & 0.2298 \\
\hline $\mathbf{4 0}$ & 0.3897 & 0.3944 & 0.3505 \\
\hline $\mathbf{5 0}$ & 0.4998 & 0.3793 & 0.4651 \\
\hline $\mathbf{6 0}$ & 0.6859 & 0.5004 & 0.5753 \\
\hline $\mathbf{7 0}$ & 0.8165 & 0.7049 & 0.7466 \\
\hline $\mathbf{8 0}$ & 0.7993 & 0.8476 & 0.7972 \\
\hline $\mathbf{9 0}$ & 0.8994 & 0.9250 & 0.9167 \\
\hline $\mathbf{1 0 0}$ & 1.0024 & 0.8966 & 0.910 \\
\hline
\end{tabular}

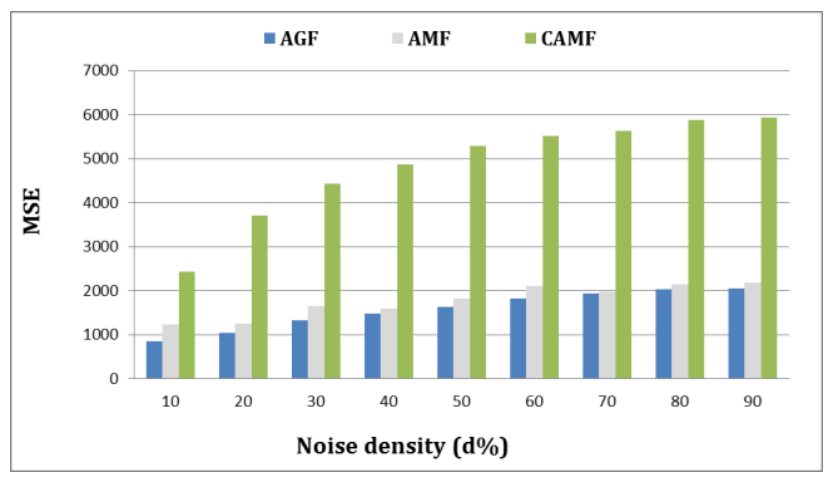

Figure 8: MSE versus of gaussian noise.

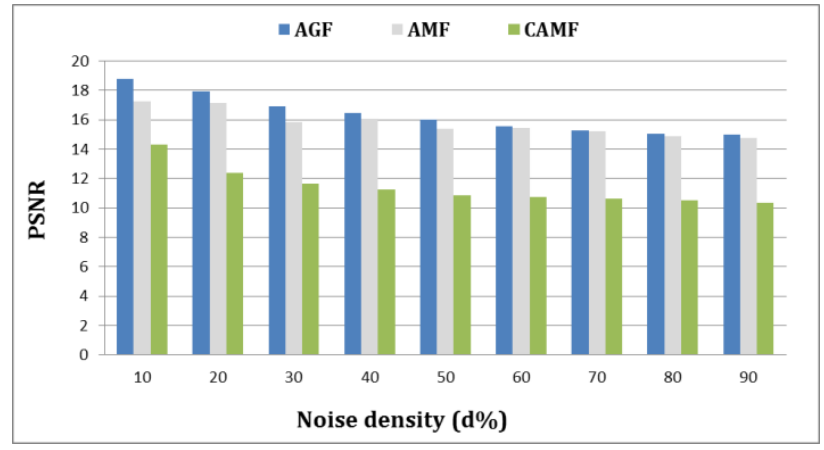

Figure 9: PSNR versus of gaussian noise.

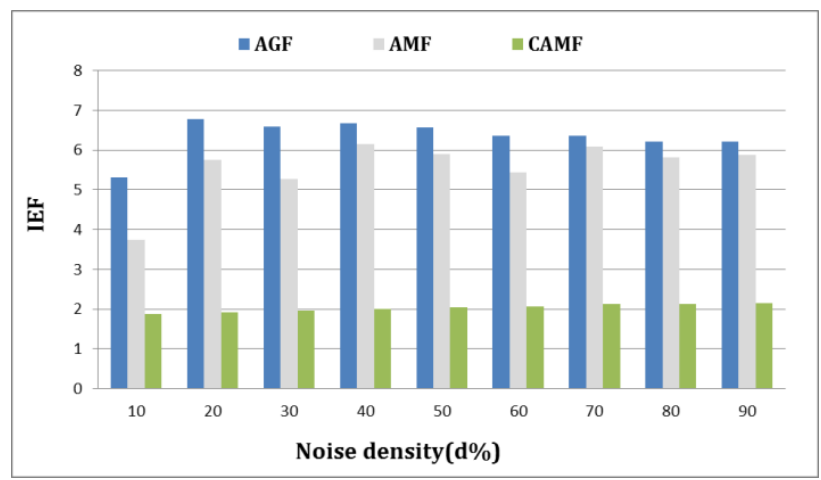

Figure 10: IEF versus of gaussian noise.

As for Gaussian noise, AGF filter exhibits the most optimum results through the whole range of noise density from 10\%-90\%. This is demonstrated by the average values of the MSE, PSNR and IEF quality metrics, which are 1576.296, 16.3231 and 6.3398 , respectively. AGF filter is followed by AMF filter with average values of $\mathrm{MSE}=1772.156, \mathrm{PSNR}=15.7887$ and $\mathrm{IEF}=$ 5.5602. The worst outcomes are attained by CAMF filter of $\mathrm{MSE}=4855.889, \mathrm{PSNR}=11.4112$ and $\mathrm{IEF}=2.0358$.

All measures and images show exceptional performance for filters through the noise density range of less than $30 \%$. However, performance is gradually getting less and less as the noise density percentage increases more than $30 \%$, This is in addition to some blurring occurred in this range, taking into consideration that the noisy image information is getting less and less in the range greater than $30 \%$. 

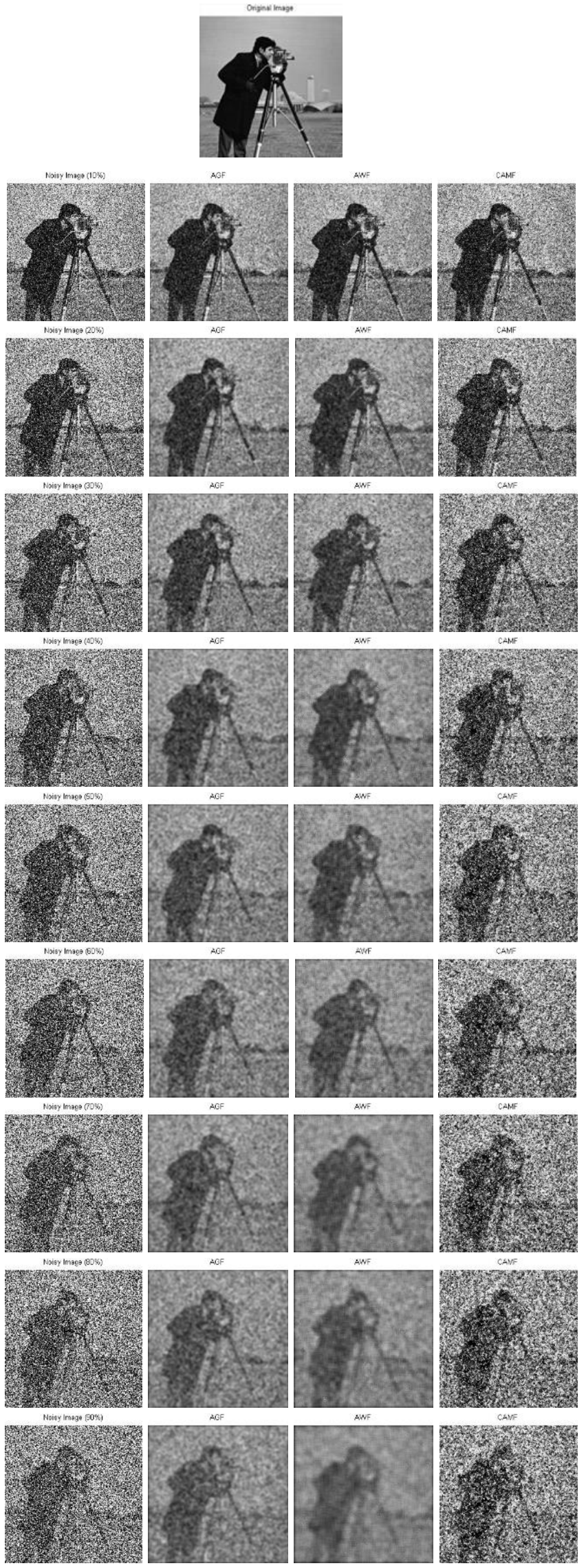

Figure 10: Processed results of various AFs for image corrupted by Gaussian noise.

\section{Conclusions}

This study is conducted to bridge the digital image processing (DIP) with Artificial Intelligence. The study was successful within its proposed assumptions and objectives. The ANN was able to estimate the noise intensity inherited in the image signal, which was utilized accordingly by filters in restoring the best possible intelligent image. The ANN showed reliable performance in estimating the noisy image signals throughout the noise density range from $10 \%$ to $100 \%$. In its turn, this enabled adaptive filter to determine its suitable variables precisely.

When the average performance is taken, improvement rates confirm that the compound image restoration system is robust. In the Salt \& Pepper noise density range of 10\%-30\%, ASMF is the best with an average of performance improvement rate equals to 97\%. CAMF comes next with an average of improvement rate equals to $97 \%$. AMF comes third with an average improvement rate equals to $92 \%$. While in the noise density range of $40-90 \%$, CAMF comes first with a performance improvement rate equals to $93 \%$. ASMF comes next with an average improvement rate equals to $87 \%$. AMF comes last with an average of improvement rate equals to $84 \%$.

As for Gaussian noise and via the whole range of noise density from $10 \%-90 \%$, AGF has the top performance with an average improvement rate equals to $83 \%$. AMF follows AGF with an average improvement rate equals to $81 \%$. CAMF comes far below its counter parts, with an average improvement rate equals to $50 \%$.

The overall outcomes improvement acquired in the case of Salt \& Pepper noise is much superior than that obtained in the case of Gaussian noise. This type of assessment involves both the estimated results out of the ANN and the outputs of the adaptive filter. The accomplishment alteration between the two cases can be due to the complex characteristics of Gaussian noise versus its counterpart, Salt \& Pepper

The neural network technology has taken the lead in enhancing many applications because mainly of its ability to imitate human thinking in resolving related problems. The author has admired and achieved an exceptional knowledge in this regard.

\section{REFERENCES}

[1] R. C. Gonzalez, and R. E. Woods , "Digital Image Processing", Third Edition, Pearson Prentice Hall, 2008.

[2] R. C. Gonzalez, and R. E. Woods and S. L. Eddins, "Digital Image Processing Using MATLAB". Gatesmark, Second Edition, 2009.

[3] H. Ibrahim, NSP. Kong, and TF. Ng. "Simple adaptive median filter for the removal of impulse noise from highly corrupted images". IEEE Transactions on Consumer Electronics, ,pp, 1920-1927, 2008.

[4] X. Deng, Y. Xiong, and H. Peng. "A new kind of weighted median filtering algorithm used for image Processing". In: 2008 International Symposium on Information Science and Engineering. IEEE, Vo1. 18, pp. 738-743, 2008.

[5] S. Akkoul, R. Ledee, and R Leconge. "A new adaptive switching median filter". IEEE Signal Processing Letters, Vol. 20, pp,.587-590, 2010.

[6] J. Jiang, and J. Shen. "An Effective Adaptive Median Filter Algorithm for Removing Salt \& Pepper Noise in Images". In: 2010 Symposium on Photonics and Optoelectronics. vol. 27, pp. 1-4, 2010.

[7] PG. Scholar. "Impulse Noise Removal Based on Artificial Neural Network Classification with Weighted Median Filter", International Conference on Advancements in Computing Technologies, 2018. 
[8] S.A. Kumar, P. Shukla, S.R Biradar, and S. Tiwari . "Multiclass Noisy Image Classification Based on Optimal Threshold and Neighboring Window Denoising.", International Journal of Computer Engineering Science, Vol. 4 Issue 3, 2014.

[9] K. Anil. "A review on various filtering techniques in image processing", National Journal of Multidisciplinary Research and Development, Volume 3; Issue 1; January 2018.

[10] Kim, Dai-Gyoung, et al. "Mixed Noise Removal Using Adaptive Median Based Non-Local Rank Minimization." IEEE Access 9 (2020): 6438-6452.

[11] A. Mason, J. Rioux, et al. "Comparison of objective image quality metrics to expert radiologists' scoring of diagnostic quality of MR images." IEEE transactions on medical imaging 39.4 (2019): 1064-1072.Authors.

[12] A. Addeeb and A. Aburas " DSP Filters and Quality Metrics for Image Denoising." International Journal of Scientific and Research Publications, (IJSRP), March 2019.

\section{AUTHORS}

First Author - Ahmed Abdualah Aburas, Assistant Lecturer, Elmergeb Univ, ECE Dept, Alkhoms - Libya, amaburas@elmergib.edu.ly

Second Author - Abdussalam A. Addeeb, Ph.D., Elmergeb Univ., E Assoc. Prof, ECE Dept, Alkhoms - Libya, dr.aaddeeb@gmail.com

Correspondence Author - Ahmed Abdualah Aburas, amaburas@elmergib.edu.ly, 218-911136459. 